Mojca Smolej* UDK [811.163.6'373.4:355.01"1791/1800"]:811.133.1

Université de Ljubljana

DOI: $10.4312 /$ linguistica.58.1.23-32

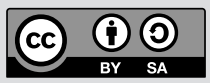

\title{
« BONAPARTE NE CHEVAUCHAIT EN PERSONNE ENTRE LES LIGNES DE TIR ET LES TROUPES ET ATTISAIT LE COURAGE DANS LES CQEUR ». L'INFLUENCE DU FRANÇAIS SUR LE VOCABULAIRE MILITAIRE DANS LJUBLJANSKE NOVICE DE VALENTIN VODNIK
}

\section{INTRODUCTION}

Les premiers écrits slovènes ayant trait à l'armée et aux actions militaires apparaissent à la fin du XVIII et au début du XIX ${ }^{\mathrm{e}}$ siècle sous la plume de Valentin Vodnik qui informe les lecteurs des événements militaires ayant lieu dans le monde colonial et décrit avec beaucoup de précision les événements concernant les guerres napoléoniennes.

En dehors des descriptions de batailles influencées par le style poétique, il faut impérativement noter que le vocabulaire militaire utilisé par Vodnik est en grande majorité d'origine allemande ou française. Dans la présente contribution, nous nous intéresserons en priorité au vocabulaire d'origine française. Comme il est difficile de déterminer si nous avons affaire ici à des jargonismes, des termes ou des mots et expressions relevant du vocabulaire général, nous comparerons le vocabulaire militaire de Vodnik au vocabulaire inclus dans le premier dictionnaire militaire slovène, rédigé en 1873 par Andrej Komel.

\section{LE BIHEBDOMADAIRE LJUBLJANSKE NOVICE}

Valentin Vodnik ${ }^{1}$ commença à publier le premier journal en langue slovène le 4 janvier 1797. Vodnik reprend la majorité des nouvelles qu'il diffuse du journal viennois Wiener Zeitung. En dehors des nouvelles concernant Vienne, il publie aussi les nouvelles concernant les événements politiques et militaires provenant des autres pays européens (par exemple, les guerre napoléoniennes) ou du monde colonial. Quand, en mars 1797, les Français traversèrent le territoire slovène Vodnik plaça régulièrement au tout début

* mojca.smolej@ff.uni-lj.si

1 Valentin Vodnik est né le 3 février 1758 dans une famille des faubourgs de Ljubljana. Pendant les années 1769-1775, il fréquenta l'école latine. Une fois sa scolarité terminée, il entra dans l'ordre franciscain. Il poursuivit tout sa vie avec persévérance sa formation intellectuelle par l'étude de l'italien et le français. En 1798, il fut nommé professeur au lycée de Ljubljana. La création des Provinces illyriennes en 1809 eut une très grande influence sur l'œuvre de Vodnik. En 1811, il publia un poème intitulé « L'Illyrie ressuscitée » (« Ilirija oživljena ») qui lui valut plus tard d'être accusé de francophilie par les autorités autrichiennes. Les autorités françaises nommèrent Vodnik professeur de français et il devint même, en 1810, proviseur du lycée qui venait d'être réorganisé. Après le retour des Autrichiens au pouvoir, Vodnik dut prendre sa retraite et cesser son activité. Il mourut en janvier 1819. 
du journal les nouvelles concernant la conquête français (voir notamment les numéros $25,26,27,28)$. Par la suite, Vodnik consacre des numéros entiers à la situation militaire et à la publication des avis rendus publics par les Français et que les lecteurs peuvent ainsi lire en slovène (voire les numéros 31, 32, 33, 34, 38, 39, 40, 41, 42).

Deux mentions de Napoléon

Bonaparte je drugi dan v'nedelo ob defetih dalej fhel s' vezh generalmi, inu kojniki, kakor menimo v' Gorizo. Njega vos je fheft kojn vosilo. Kashe, de ni popolnoma sdrav; on fe je tukej v' morji kopal. (LN 1797, n. 38) ${ }^{2}$

Sam Bonaparte je hitil k'rafdelo Lasues, de bi bil negovo beshanje vftavil, sato, kir fhe pomagavna truma generala Pefaix ni perpravlena bila. Bonaparte je fam fovrashnika snegovimi sagrabiti otel ali ufaki tega mofhtva je upil, pervi Konsul naj nehodi v' nevarnoft. Negovi foldatje fo bili ket bi ih bil podbodil, fo posabili na vfo nevarnoft, sato kir lo nih perviga napelvalza tako ferzhniga vidili. (LN 1800, n. 31)

Les Novice de Vodnik cessèrent de paraître au bout de quatre ans. Parmi les raisons diverses qui conduisirent à cette disparition, il y eut avant tout la censure autrichienne, mais aussi les difficultés financières, le peu d'abonnés, les liaisons postales irrégulières et la concurrence de la presse allemande.

\subsection{La langue populaire et poétique}

Avant d'examiner le vocabulaire militaire, nous nous concentrerons sur la définition sociale et fonctionnelle de la langue dans le journal Ljubljanske novice. ${ }^{4}$

Bien que nous ayons affaire à un texte journalistique, nous ne pouvons pas qualifier la langue de Vodnik comme relevant d'un style journalistique entièrement développé.

Ainsi, dans $L N$ nous remarquons de nombreuses composantes linguistiques populaires et poétiques qui témoignent du talent de Vodnik comme écrivain et poète. Les nouvelles concernant les combats et la thématique militaire sont ainsi fortement empreintes d'expressivité et d'expressions populaires.

Descriptions de batailles et de sujets en rapport avec l'armée écrites dans une langue populaire et expressive :

Sam Bonaparte je jesdil med ftrelam med trumami, inu je foldate v'ferza vshigal. To je bilo ob 4ih popoldan. General Desaix je fvoje naprej napelval v'fovrashnika naravnoft, kateriga so negovi foldatje s'pufhnim fhpizami bodli. Oftanik rafdela Boudet je ravno to delal, ja fdaj je zela armada v' 2 raifah proti fovrashniki fhla. Sovrashnika pefhzi inu fhtukarji fo fe mogli nasaj vmikati. Generala Desaix sadene

2 Dans l'extrait, Vodnik parle du voyage de Napoléon à Gorizia.

3 Dans l'extrait, Vodnik parle de Napoléon qui encourage ses troupes au péril de sa vie.

4 Dans la suite de l'article : LN. 
kugla, negova fmert podshge armado fhe bel, ona fe vershe notri v'vkupsmusnene raife fovrashnika, katere fo pufhne fhpize nam naftavile, inu fo naf nasaj dershale, $v^{\prime}$ tim j e general kojnftva eno lukno sasnal, rukne fkosi inu vjame 6000 fovrashnika, med katerimi je general Zah inu S. Julien. (LN 1800, n. 31)

Les syntagmes (soulignés dans l'exemple) jezditi med strelami, soldate $v$ srca vžigati, s puškinimi špicami bosti, smrt podžge armado et vreči se v sovražnika montrent que la description de la bataille est expressive et rédigée à l'aide d'un vocabulaire général et populaire.

Et nous pourrions citer beaucoup d'exemples du même type ou'on trouve un lexique trop connoté ou populaire. Les nouvelles concernant l'armée et les combats proposées par Vodnik ne correspondent pas aux critères de la langue spécialisée. Les syntagmes poétiques (par exemple : meč je že bil potegnen, kri je imela gnojiti nemške, tirolske inu laške zemle, cesar bode kakor miru Angel nazaj prišel) sont constamment associés à des expressions populaires ou familières (par exemple : od vojske zmolzena).

À ce niveau, la recherche du jargon militaire est rendue difficile, voire impossible du fait que les seules sources de cette époque dont nous disposons sont les $L N$ de Vodnik que nous avons analysées.

Comme nous l'avons déjà dit, en dehors de l'élément populaire, nous pouvons également trouver dans les descriptions de Vodnik des éléments narratifs au moyen desquels l'écrivain accroit la tension chez le lecteur. L'expressivité se traduit non seulement à l'aide du vocabulaire, mais aussi par l'usage d'éléments grammaticaux spécifiques comme le temps verbal.

Generala Desaix sadene kugla, negova Smert podshge armado fhe bel, ona fe vershe notri v'vkupsmusnene raife fovrashnika, katere fo pufhne fhpize nam naftavile, inu fo naf nasaj dershale, v'tim je general kojnftva eno lukno sasnal, rukne Skosi inu vjame 6000 fovrashnika, med katerimi je general Zah inu S. Julien. General Lasnes gre potim s' rasdflami Watrin, Bondet, jesdezhim granadirji inu fhtuki, inu kojniki kateri fo kojnike fovrashnika popadli inu ih nasaj sagnali; kir fmo fe she eno uro v tami mahali, fovrashnik je veliko vezh vjetih, oftrelenih inu pobitih fhtel pravi Bertier. Tako fe je končal leta imenitni boj od kakerfhniga na Lafhkim ni flifhati bilo, koker od Iejta 1525 per Pavii. (LN 1800, n. 31)

Par l'utilisation du présent historique (souligné dans le texte), la prédominance de l'asyndète et le recours au vocabulaire populaire (par exemple : smo se mahali, rukne skozi), la description de Vodnik s'éloigne de la communication objective propre aux journalistes. En revanche, elle se rapproche considérablement des caractéristiques de la langue littéraire.

5 Les passages soulignés utilisent les expressions comme chevaucher parmi les foudres, attiser le courage dans les cœurs, charger à la baïonnette, la mort qui enflamme les soldats, se jeter sur l'ennemi.

6 Dans l'extrait, Vodnik décrit les événements qui ont suivi la mort du général Desaix. 


\subsection{Le vocabulaire militaire}

En traduisant les nouvelles militaires publiées dans le quotidien viennois Wiener Zeitung, Vodnik était constamment gêné par l'absence des expressions militaires slovènes qui lui étaient nécessaires. L'analyse des mots et expressions utilisés par Vodnik montre que l'auteur a eu recours à quatre procédés distincts pour élaborer un vocabulaire adéquat.

Il a pu trouver une partie du vocabulaire dans les sources déjà existantes (par exemple, la Bible). ${ }^{7}$

En effet, Vodnik rencontrait également le vocabulaire militaire en tant que traducteurs des proclamations officielles. Nous savons que ce type de documents commencèrent à se traduire et à s'imprimer en slovène à l'époque de Marie Thérèse.

Pour traduire les nouvelles militaires, Vodnik a recours à des mots et expressions reprises des langues étrangères, le plus souvent de l'allemand, du français et de l'italien, et ce avec différents degrés d'adaptation à la langue slovène. Parfois, il a opté pour des calques trouvés dans la langue populaire (par exemple, kugle, špegavci).

Pour le vocabulaire d'origine française ou italienne, il est assez souvent difficile de déterminer si l'écrivain slovène les a repris directement ou par l'intermédiaire de la langue allemande.

Dans la suite de notre présentation, nous allons nous concentrer sur le vocabulaire d'origine française sans nous demander si celui-ci a été repris directement ou indirectement par l'intermédiaire de l'allemand.

\subsubsection{Le vocabulaire militaire repris du français ${ }^{8}$}

Tout d'abord, il convient de mentionner que nous évitons volontairement de parler de « terminologie », du fait que tout le vocabulaire étudié a été écrit dans le journal Ljubljanske novice, donc dans des textes destinés au grand public. Les débuts de l'élaboration de la terminologie militaire slovène, en réalité, ne remontent pas avant 1872, année de parution du petit dictionnaire slovène-allemand et allemandslovène intitulé Poljna služba, raztreseni red, nova puška in slovensko-nemški in nemško-slovenski slovarček d'Andrej Komel. À la fin de cette présentation des expressions militaires de Vodnik, nous les comparerons brièvement avec la langue militaire de Komel.

Dans le vocabulaire potentiellement repris du français, nous pouvons remarquer aussi bien des emprunts que des mots étrangers adaptés et cités.

7 Dans la Bible (et déjà dans la langue des écrivains protestants), le vocabulaire militaire a été formé soit sur la base d'un vocabulaire général et populaire d'origine slovène (boj, orožje, bojevati se, bramba, vojskovati se, pešec, kojnik, ukazati...) ou bien sur la base d'une influence allemande ou latine (kamp, legion, gliha ...).

8 La section 2.2.1. a été rédigée grâce à la consultation et à l'analyse des Ljubljanske novice mais aussi de la recherche présentée en 1995 par Andreja Legan-Ravnikar sous le titre Valentin Vodnik - oblikovalec slovenske terminologije (Upravno-politična in vojaška terminologija, Lublanske novice, 1979), plus particulièrement des pages 134 à 168 . 


\subsubsection{Les emprunts}

Un emprunt est un mot venu d'une autre langue et totalement adapté aux éléments constitutifs de la langue slovène qui l'intègre.

$\begin{array}{lll}\text { Emprunt dans } \boldsymbol{L N} & \text { Équivalent français } & \text { Équivalent allemand } \\ \text { Bomba } & \text { bombe } & \text { Bombe } \\ \text { baterija, batteria } & \text { batterie } & \text { Batterie } \\ \text { Garnison } & \text { garnison } & \text { Garnison } \\ \text { grenater, grenatjer, granadier } & \text { grenadier } & \text { Grenadier } \\ \text { eskadron, Escadron } & \text { escadron (de l'it. squadrone) } & / \\ \text { Kokarda } & \text { cocarde } & / \\ \text { komanda, commanda } & \text { commande } & \text { Kommando } \\ \text { komendant, commandant, } & \text { commandant } & \text { Kommandant } \\ \text { comandant, komendant, } & & \\ \text { komentant } & & \\ \text { korporal } & \text { caporal } & \text { Korporal } \\ \text { Rekrut } & \text { recrue } & \text { Rekrut } \\ \text { Brigada } & \text { brigade } & \text { Brigade } \\ \text { Admiral } & \text { admiral } & \text { Admiral } \\ \text { Šalupe } & \text { chaloupe } & \text { / } \\ \text { Parada } & \text { parade } & \text { Parade } \\ \text { Patron } & \text { patron } & \text { Patron } \\ \text { Marš } & \text { marche } & \text { Marsch } \\ \text { regiment } & \text { regiment } & \text { Regiment } \\ \text { Kavaler } & \text { cavalier (de l'it. cavaliere) } & \text { / } \\ \text { kvarter, kvartier, quartier } & \text { quartier } & \text { Quartier }\end{array}$

\subsubsection{Les mots étrangers adaptés et cités}

L'examen des mots étrangers montre que Vodnik les a le plus souvent également repris par l'intermédiaire de l'allemand.

Un mot étranger adapté (tujka) est un mot partiellement adapté à la langue slovène, tandis que le mot étranger cité (citirana beseda) conserve sa forme d'origine.

$\begin{array}{lll}\text { Mot étranger dans LN } & \text { Équivalent français } & \text { Équivalent allemand } \\ \text { korvetta, korveta, corvetta } & \text { corvette } & \text { Korvette } \\ \text { batallion, batalion, battallion } & \text { bataillon } & \text { Bataillon } \\ \text { Artilleria } & \text { artillerie } & \text { Artillerie } \\ \text { Terrorist } & \text { terroriste } & \text { Terrorist } \\ \text { flotta, flota } & \text { flotte } & \text { Flotte } \\ \text { kompania, kompanija } & \text { compagnie } & \text { Kompanie } \\ \text { Division } & \text { division } & \text { Division } \\ \text { patrulla, patrolla } & \text { patrouille } & \text { Patrouille } \\ \text { kurier, couriere } & \text { courier } & \text { Kurier } \\ \text { general-gouverneur } & \text { gouvereur } & \text { Gouverneur } \\ \text { Leutenant } & \text { lieutenant } & \text { Leutnant } \\ \text { Dragonar } & \text { dragon } & \text { Dragoner } \\ \text { Kanonada } & \text { canonnade } & \text { Kanonade } \\ \text { reserve armada } & \text { reserve } & /\end{array}$


La relative incohérence des écrits, visible dans l'existence de doublons, voire de triplons, est quelque chose d'habituel si on considère que la norme régissant l'écriture slovène n'a commencé à s'arrêter qu'à partir de 1851, voire 1854, quand est parue la grammaire de Janežič.

Il convient une fois encore que les expressions militaires mentionnées ici, sont utilisées dans la presse, ce qui signifie que certaines expressions peuvent avoir revêtu une signification différente de celle qu'elles avaient dans la langue originale.

Dans la suite de la présente contribution, nous comparerons le vocabulaire utilisé par Vodnik, celui d'Andrej Komel et la terminologie militaire contemporaine.

\subsubsection{Comparaison entre le vocabulaire d'Andrej Komel ${ }^{9}$ et celui du diction- naire des expressions militaires contemporain}

Nous pourrons ensuite comparer ces deux sources avec le dictionnaire militaire slovène contemporain paru en 2002. Comme ni le dictionnaire de Komel ni le Dictionnaire militaire de 2002 ne fournissent d'explications concernant la signification des expressions militaires choisies, de même qu'il nous est impossible de fournir des explications sémantiques complètes concernant les expressions militaires utilisées par Vodnik dans les $L N$, nous pouvons seulement émettre des suppositions sur l'existence d'un processus de déterminologisation et de reterminologisation.

\begin{tabular}{|c|c|c|}
\hline$L N(1797-1800)$ & A. Komel (21873) & Dictionnaire militaire (2002) \\
\hline Admiral & admiral & admiral \\
\hline Artilleria & l & artilerija \\
\hline batallion, batalion, battallion & bataljon & bataljon \\
\hline baterija, batteria & baterija, topovnica & baterija \\
\hline Bomba & / (bombardovanje) & bomba \\
\hline Brigada & brigada & brigada \\
\hline Division & divizija & divizija \\
\hline Dragonar & l & I \\
\hline eskadron, escadron & I & eskasdron (zgod.) \\
\hline flotta, flota & / & flota \\
\hline Garnison & posadka & garnizija \\
\hline grenater, grenatjer, granadier & / (deželni noolavar) & ' (oeneral) \\
\hline $\begin{array}{l}\text { Kavaler } \\
\text { Kall }\end{array}$ & / (aezenin pograval) & / \\
\hline Karronada & / & I \\
\hline Kokarda & l & 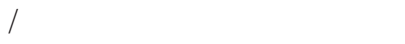 \\
\hline komanda, commanda & $\begin{array}{l}\text { povelje, poveljništvo, } \\
\text { (veleti, zapovedovati) }\end{array}$ & poveljništvo \\
\hline $\begin{array}{l}\text { komendant, commandant, } \\
\text { comandant, komendant, } \\
\text { komentant }\end{array}$ & poveljnik & poveljnik \\
\hline Korporal & / & korporal (zgod.) \\
\hline
\end{tabular}

9 Pour analyser le dictionnaire de Komel, nous avons également consulté le dictionnaire de Tomo Korošec, Slovenski vojaški jezik (1998). 


$\begin{array}{lll}\boldsymbol{L N}(\mathbf{1 7 9 7 - 1 8 0 0 )} & \text { A. Komel (21873) } & \text { Dictionnaire militaire (2002) } \\ \text { kompania, kompanija } & / & \text { kompanija (zgod.) } \\ \text { korvetta, korveta, corvetta } & / & \text { korveta } \\ \text { kurier, couriere } & / & \text { kurir } \\ \text { kvarter, kvartier, quartier } & / & / \\ \text { Leutenant } & / & \text { (generallajtnant (zgod.)) } \\ \text { Marš } & \text { marš, hod, pohod } & \text { pohod } \\ \text { Parada } & / & \text { parada } \\ \text { Patron } & \text { naboj, strel, patrona } & \text { naboj (patron) } \\ \text { patrulla, patrolla } & \text { patrola, obhodna straža } & \text { patrulja, obhodna patrulja } \\ \text { Regiment } & / & / \\ \text { Rekrut } & / & \text { rekrut } \\ \text { reserve armada } & \text { založna armada, reserva } & \text { rezervna armada } \\ \text { Šalupe } & / & / \\ \text { Terrorist } & / & \text { terorist }\end{array}$

En observant cette liste, nous pouvons distinguer trois processus évolutifs distincts dans la formation du vocabulaire militaire d'origine française. Le plus fréquent est la conservation du mot repris qui figurera ensuite parmi les termes standards contemporains (artilerija, bataljon, brigada, divizija...). Dans le second groupe se trouvent les mots connotés temporellement et se voyant, de ce fait, qualifiés d'historiques par le qualificateur zgod. (korporal, kompanija, eskadron, ...). Dans le troisième groupe, qui est peut-être le plus intéressant, se trouvent les expressions qui ont été déplacées du registre standard ou littéraire vers le registre du jargon militaire (komandant, marš et patron). Pour tous ces mots, il existe une expression standard ou littéraire équivalente au niveau sémantique (poveljništvo, poveljnik, pohod et naboj).

À l'intérieur du premier groupe, celui des mots déjà employés par Vodnik et demeurant encore dans le dictionnaire militaire contemporain, il y a également deux mots pour lesquels Komel propose des équivalents slovènes qui sont oubliés aujourd'hui : 《topovnica » pour « baterija » et " posadka » pour " garnizija ». La terminologie militaire contemporaine les a rejetés, car ils ne sont pas des synonymes parfaits des mots d'origine étrangère.

\section{EN GUISE DE CONCLUSION}

Étant donné le peu de documents dont nous disposons (notamment du peu de lettres privées envoyées par les soldats), nous pouvons difficilement parler du jargon militaire slovène durant la seconde moitié du $\mathrm{XIX}^{\mathrm{e}}$ siècle et la première moitié du $\mathrm{XX}^{\mathrm{e}}$ siècle. En revanche, nous pouvons pleinement parler d'une langue militaire portant toutes les caractéristiques de l'utilisation expressive qui en était faite. Il s'agit d'une manière poétique ou, du moins, littéraire d'aborder la thématique militaire. Le principal transcripteur de cette langue fut précisément Valentin Vodnik. ${ }^{10}$

10 C'est ce dont témoigne, entre autres, le passage suivant : "Sovrashnik fe fdaj s tremi veliki rafdeli proti nam blisha, inu sagrabi trumo Viktor s' vfo mozhjo, katera fe je proti Seravale potegnila, Bertier je sdaj rafdelo Desaix sapovedal naprej riniti inu je hitel s' njo s' Bonapartam $\mathrm{v}$ boj, on najde pretepajne inu zofedranie s'sablami inu pufhami poglavanje. General Gardane fe 


\section{Bibliographie}

KOMEL, Andrej ('1873) Bojna služba, raztreseno vojevanje, nova puška in slovenskonemški in nemško-slovenski slovarček. Celovec : Družba sv. Mohora.

KOROŠEC, Tomo (1998) Slovenski vojaški jezik. Ljubljana : Fakulteta za družbene vede

KOROŠEC, Tomo (2007) «O Komelovih naslovnih vprašalnih stavkih nasproti nemškim samostalniškim poimenovanjem. »In : Irena Orel (éd.), Razvoj slovenskega strokovnega jezika. Ljubljana : Filozofska fakulteta, Oddelek za slovenistiko, Center za slovenščino kot drugi/tuji jezik, 201-207.

KOROŠEC Tomo Korošec et al. (éds) (1977) Vojaški slovar. Ljubljana : Partizanska knjiga.

KOROŠEC Tomo et al. (éds) (2002) Vojaški slovar. Ljubljana : ministrstvo za obrambo LEGAN RAVNIKAR, Andreja (1995) Valentin Vodnik - oblikovalec slovenske terminologije : (upravno-politična in vojaška terminologija - Lublanske novice 1797) : magistrska naloga. Ljubljana : Oddelek za slovanske jezike in književnosti, Filozofska fakulteta $v$ Ljubljani.

OROŽEN, Martina (1985) « Smernice knjižnega jezikovnega razvoja od Jurija Dalmatina do Jurija Japlja (1584-1784). » Jezik in slovstvo 30/7, 217-223.

OROŽEN, Martina (1991) « Odmevi francoske revolucije v Vodnikovih Novicah : 1797-1800. » In : Matjaž Kmecl (éd.), Obdobje slovenskega narodnega preporoda. Ljubljana: Filozofska fakulteta, Oddelek za slavistiko, 195-208.

OROŽEN, Martina (1989) « Napoleonov razglas za Kranjce : (ljubljanski jezikovni različici prevoda iz francoščine in nemščine). » In : Breda Pogorelec (éd.), Jezikoslovne in literarnovedne raziskave : zbornik referatov 6. srečanja slavistov CelovecLjubljana. Ljubljana : Filozofska fakulteta, 67-85.

Slovar slovenskega knjižnega jezika. 2. Avril-Octobre 2016. http://www.sskj2.si/ uporabnik/prijava.

Sveto pismo Stare in Nove zaveze : slovenski standardni prevod iz izirnih jezikov (1996). Trad. Jože Krašovec et al. Ljubljana : Svetopisemska družba Slovenije. VODNIK, Valentin (éd.) (1797-1800) Lublanske novice. Ljubljana : J. Fr. Eger.

je she 2 uri, ne de bi bil fe ifpremiknil s'fovrashnikam na desni mahal. /... Sovrashnik je potim tolkain hujfhi v naf fe sarofil, kir fo nafhi urjeni ftrelizi she ufe iftrelali, inu ki fo fmefhani if pobijalifha beshati mogli /.../ Bonaparte je fam fovrashnika s negovimi sagrabiti otel ali ufaki tega mofhtva je upil, pervi Konsul naj nehodi v' nevarnoft. Negovi foldatje fo bili ket bi ih bil podbodil, fo posabili na vfo nevarnoft, sato kir so nih perviga napelvalza tako ferzhniga vidili » (LN 1800, n. 31). 
Résumé

«BONAPARTE NE CHEVAUCHAIT EN PERSONNE ENTRE LES LIGNES DE TIR ET LES TROUPES ET ATTISAIT LE COURAGE DANS LES CEEURS ». L'INFLUENCE DU FRANÇAIS SUR LE VOCABULAIRE MILITAIRE DANS LJUBLJANSKE NOVICE DE VALENTIN VODNIK

Dans la présente contribution, nous étudierons plus spécialement l'influence de la langue française sur l'usage du vocabulaire militaire dans Ljubljanske novice (Les Nouvelles de Ljubljana, 1797-1800) de Valentin Vodnik. L'analyse du vocabulaire militaire montre que Vodnik a construit les fondements de la terminologie militaire slovène, mais il faut souligner que nous sommes en présence d'articles de journaux ayant une autre fonction et poursuivant d'autres objectifs que les textes techniques strictement militaires. Dans les Ljubljanske novice, Vodnik décrit la situation militaire en Europe à la fin du XVIII ${ }^{e}$ siècle, notamment les guerres napoléoniennes, à l'aide de descriptions et de nombreux exemples. Pour élaborer la terminologie militaire slovène, Vodnik a souvent opté pour un emprunt aux langues étrangères, en priorité au français, ce qui fera plus spécialement l'objet de notre attention. Dans le vocabulaire repris $\mathrm{du}$ français, nous pouvons remarquer aussi bien des emprunts que des mots étrangers adaptés et cités. À la fin de cette présentation des expressions militaires de Vodnik, nous les comparerons brièvement avec la langue militaire de Andrej Komel. Comme le dictionnaire de Komel est considéré comme le fondement de la terminologie militaire slovène, nous allons regarder combien d'expressions militaires reprises du français par l'intermédiaire de l'allemand utilisées par Vodnik ont subsisté et ont été incluses dans le dictionnaire de Andrej Komel.

Mots-clés: Valentin Vodnik, Ljubljanske novice, vocabulaire militaire, influence du français

\section{Abstract \\ "BONAPARTE NE CHEVAUCHAIT EN PERSONNE ENTRE LES LIGNES DE TIR ET LES TROUPES ET ATTISAIT LE COURAGE DANS LES CEURS". THE INFLUENCE OF FRENCH ON THE MILITARY VOCABULARY OF VODNIK'S LJUBLJANSKE NOVICE}

In this article we focus on the influence of French on the military vocabulary of Lublanske novice (Ljubljana News; 1791 - 1800), the first Slovene newspaper. The analysis reveals that in the articles published in this newspaper Valentin Vodnik laid the foundation of Slovene military terminology. He reported on military events from the colonial world and accurately cited developments during the Napoleonic Wars. Vodnik's military language is untypical in that it is replete with expressive words and phrases as well as with various rhetorical figures, and as such has a literary or poetic quality. In addition to the somewhat poetic descriptions of warfare, it is necessary to 
draw attention to Vodnik's military vocabulary which is mostly of German and French origin. However, in the present article, we focus only on the vocabulary which could have came into Slovene from French (possibly via German). Since it is difficult to find out with Vodnik's articles whether we are dealing with jargonisms, terms or general lexis, we compare his military vocabulary with that of the first Slovene military dictionary compiled by Andrej Komel in 1872. The comparison shows to what extent Vodnik's military vocabulary was taken over into Slovene military terminology, as far as it can be gathered from Komel's work.

Keywords: Valentin Vodnik, Lublanske novice, military vocabulary, influence of French

Povzetek

»SAM BONAPARTE JE JEZDIL MED STRELAM MED TRUMAMI, INU JE SOLDATE V SRCA VŽIGAL.« VPLIV FRANCOŠČINE NA VOJAŠKO BESEDIŠČE VODNIKOVIH LJUBLJANSKIH NOVIC

$\mathrm{V}$ pričujočem prispevku se osredotočamo na vpliv francoščine na vojaško besedišče v Ljubljanskih novicah (1791-1800). Analiza besedišča razkriva, da je Valentin Vodnik oblikoval temelje slovenski vojaški terminologiji. Vodnik je namreč poročal o vojaških dogodkih iz kolonialnega sveta, natančno pa je popisoval dogajanja za časa Napoleonove vojne. Vodnikov vojaški jezik je poseben, saj je napolnjen z ekspresijo, najrazličnejšimi retoričnimi figurami in se kot tak približuje literarno oz. pesniško obarvani dikciji. Poleg pesniško obarvanega opisa bojevanj pa je pri Vodniku nujno opozoriti tudi na samo vojaško besedišče, ki je povečini nemškega in francoskega izvora. $\mathrm{V}$ pričujočem prispevku se osredotočamo le na besedišče, ki je izvorno francosko. Ker pri Vodniku težko ugotavljamo, ali gre za žargonizme, termine ali splošno besedišče, Vodnikovo vojaško besedišče primerjamo $\mathrm{z}$ besediščem prvega slovenskega vojaškega slovarja, ki ga je 1872 sestavil Andrej Komel. S to primerjavo je vidno, kolikšen delež Vodnikovih vojaških izrazov je prešel med strokovne izraze oz. je bil v rabi še v drugi polovici 19. stoletja.

Ključne besede: Valentin Vodnik, Ljubljanske novice, vojaško besedišče, vpliv francoščine 